\title{
Das Recht als Netz für Netzwerke: Eine Wegskizze
}

Thema dieses Beitrags ist, die Begriffe »Netzwerk « und »Recht« zu einander in Bezug zu setzen. Beschrieben werden soll damit der gedankliche Vorgang der Bestimmung des Verhältnisses der beiden Größen zueinander. Der Begriff des Netzwerks übt zur Zeit eine enorme Anziehungskraft aus, und solche Anziehungskraft hat fast von selber mit seiner Weite und Offenheit zu tun. Damit ist die Frage nach dem Fassungsvermögen des Rechts auf dem Plan, und das feedback zurück zum Netzwerkbegriff ist damit absehbar als ein Stück Max Weber'scher Entzauberung.

\section{Das Recht als Orakel}

Die beiden Begriffe kennzeichnet zunächst einander gegenüber Passivität. Sie gehen einander »nichts an«, weil der eine der Welt der Erscheinungen, der andere der Welt der Normen entstammt. Die Situation ist insofern die normale im Verhältnis von Sein und Sollen.

Passiv ist - so gilt es zunächst festzuhalten - von seiner Natur her das Recht. Recht macht Aussagen, und dafür ist es zu befragen. Es steht zur Verfügung, wenn das Verhalten zwischen Einheiten (Individuen oder Organisationen) von diesen zu bestimmen ist. Was es anbietet, sind Regeln oder, etwa im Case Law, auch normative Äußerungen zu konkreten Fällen.

Diese Kennzeichnung kann sich natürlich nur auf das Recht beziehen, nicht auf den Rechts-Stab - um nochmals eine Prägung Max Webers zu verwenden. Der Rechtsstab setzt die Passivität des Rechts in Aktivität um. Beamte, Strafverfolgungsbehörden und Rechtswissenschafter etwa haben spontan »im Namen des Rechts « zu handeln.

Der Richter ist, als weiteres Glied des Rechtsstabs, das Gegenbeispiel. Er äußert sich grundsätzlich nur auf Befragung, und dabei ist er »la bouche de la loi «: er »spricht Recht«. Er hat Orakel-Funktion, während die andern Glieder des Rechtsstabs, vom Recht veranlasst werden; sie agieren, üben mit Berufung auf das Recht Herrschaft aus. Die beiden Blickweisen auf das Recht soll man nicht vorschnell mit »privat-« bzw. »öffentlichrechtlich« etikettieren; denn beide Arten von Rechtsstabsfunktionen finden sich in beiden Bereichen des materiellen Rechts.

Es gibt aber auch den Punkt, wo Information in der umgekehrten Richtung fließt. Der Rechtsstab ist auch zuständig, sich über die Bewährung des vorhandenen Rechts zu vergewissern. Alle Recht schaffenden Instanzen haben in ihrer Umwelt Umschau zu halten. Das gibt den neuen oder neu bedachten Erscheinungen die Chance, ins Recht aufgenommen zu werden. Insoweit hat zwar nicht das Recht, aber der Rechtsstab eine durchaus aktive Rolle in der Herstellung der Verbindung zu den Phänomenen.

Auch in Hinsicht auf das Recht selber ist sogleich beizufügen, dass Inaktivität nicht Insensibilität bezüglich des Neuen bedeutet. Jede Frage an das Recht ist neu, weil kein Sachverhalt mit einem andern identisch ist. Die Bindung durch vorangehende Generalisierungen ist jedenfalls nicht vollständig, wenn geltungszeitliche Auslegung, ja teleologische Reduktion möglich sind. Das Recht ist mithin offen für input jeder Art. 


\section{Die Initiative der Umwelt}

Von der andern Seite, von den Phänomenen aus gesehen, sieht es entsprechend aus. Auch diese zeigen dem Recht zunächst die »kalte Schulter«; sie sind nicht Geschöpfe des Rechts und existenziell insofern nicht von ihm abhängig. Das Recht kann aber ihrer Entfaltung Bedingungen auferlegen, ob das durch Androhung der Intervention oder dadurch geschehe, dass es über die Benutzbarkeit seiner Instrumente für die jeweiligen faktischen Ziele entscheidet, dass es also vertragliche Bindungen, Eigentumsrechte, organisatorische Einheiten und Anderes in solchen Kontexten anerkennt.

Das gibt Anlass zur Befragung des Rechts.

\section{Die Bedeutung der gewachsenen Sprache}

Die Praxis erfasst die Erscheinungen in ihrer Sprache, und formuliert darum auch ihre Probleme so. Für die Befragung des Rechts bedarf es deshalb einer Übersetzung; denn das Recht hat seinerseits seine eigene Sprache, welche für die Erschließung seiner Normbestände unabdingbar ist.

Wer immer diese Übersetzungsleistung erbringt - am ehesten der jeweils (Fakten bzw. Recht) suchende Teil -, ist es nicht die Sprache einer andern Wissenschaft, sondern einer Gemeinschaft als dem Ort, wo Bedürfnisse des praktischen Handelns entstehen. Solche Gemeinschaften - der Begriff verstanden im weitesten Sinn mit Blick sowohl auf eine geschlossene wie auch auf eine offene und große Gruppe, etwa einer nationalen oder professionellen usw. Öffentlichkeit - sind für das Recht Problemproduzenten. Ihre Sprache ist das aus der Gemeinschaft erwachsene Idiom. Wissenschaft kann dazu aber durch Anstöße und Präzision beitragen.

Die Übersetzung von einer Wissenschaft ins Recht bedarf also zweier medialer Schritte. Zum Einen muss sie eine möglichst breit verstandene »Stimme des Volks« als betroffenen Rechtsgenossen finden, und zum Andern sind von da her Rechtsregeln abzurufen oder vorzuschlagen.

Dies ist im Fall von Netzwerken für ihre Stellung im Recht von geradezu entscheidender Bedeutung. Der Ausdruck wird in den Kontexten verschiedener Disziplinen verwendet, was fast zwangsläufig auch verschiedene Bedeutungen erzeugt. Das sind schlechte Voraussetzungen für einen Terminus, der eine schier unglaubliche Konjunktur im allgemeinen Gebrauch erlebt. Der wissenschaftliche wie der allgemeinsprachliche Boom zeigen an, dass das Sinnbild des Netzwerks in einer Weise dem zeitgenössischen Ausdrucksbedürfnis entgegenkommt wie kein Begriff mehr seit dem Hochkommen desjenigen der Information.

Im allgemeinen Gebrauch verwischen sich die Konturen. Und da auch die Wissenschaften den Begriff bis dahin nur ausgesaugt, d.h. als Ausgangspunkt für eigene Forschungen verwendet, aber nicht zu seiner Präzisierung als einheitlichem Begriff beigetragen haben - ein typischer Fall von Übernutzung der Allmende -, ist sein Aussagegehalt schwach und unsicher geworden. Diese Verflachung erschwert die Aufnahme ins Recht, weil dafür Eigenschaften des Phänomens zu benennen sind, die Probleme erzeugen, welchen ihrerseits mit Rechtsregeln geantwortet werden kann. 


\section{Die begriffliche Aufnahmefähigkeit des Rechts}

Wir wenden damit den Blick wieder auf die Gegenseite. Wie reagiert das Recht auf das »Netzwerk «? Drei Stufen der Aufbereitung des Begriffs bzw. des von ihm bezeichneten Phänomens sind zu unterscheiden.

Zunächst ist es die Konfrontation: Was wird gleichsam an juristischen Programmen durch den Begriff »Netzwerk« als solchen aktiviert? Die Antwort ist einfach: gar nichts. Der Begriff ist im Recht nicht angelegt. In dem Sinn ist die in diesem Band mehrfach erwähnte Aussage von Richard Buxbaum, gewiss richtig: «>Network $<$ is not a legal concept $\varkappa^{1}$. Heben wir uns für einen Augenblick die Frage auf, ob sie eine bloß positivrechtliche Gegebenheit oder eine Denknotwendigkeit darstellt. Jedenfalls sind wir auch der Überführung des außerjuristischen Begriffs in einen juristischen enthoben.

Auch wenn die juristischen Sensoren auf den Begriff tel quel nicht angeben, kann dies der Fall sein, wenn wir ihn in seine Eigenschaften auflösen. Die rechtliche Aufgabe kann dann auf solche Eigenschaften ausgerichtet werden. Wenn wir die Vorstellungen analysieren, die gemeinhin mit einem »Netzwerk« assoziiert werden, könnte dies etwa sein:

- Die Horizontalität der Struktur

- Die Informalität

- Die Offenheit des Zugangs

- Die Nicht-Exklusivität gegenüber andern Zugehörigkeiten.

Doch betrachten wir den Gebrauch, der vom Begriff gemacht wird, so ist keine dieser Charakterisierungen zuverlässig, noch ganz abgesehen von ihrer Unbestimmtheit.

Als eine dritte Möglichkeit ließe sich dieser Pfad aber weiter verfolgen, indem das Recht im Sinn einer pars pro toto den rechtlichen Netzwerkbegriff von sich aus verengt. Das entspricht einer allgemeinen juristischen Technik. Eine Ehe im Rechtssinn setzt einen Formalakt voraus, Insiderdelikte oder ad hoc-Mitteilungpflichten sind auf feststehende Tatsachen beschränkt, ein Konzernverhältnis kennzeichnet sich durch Mehrheitsbesitz oder einheitliche Leitung: In unzähligen Konstellationen verzichtet das Recht im Sinne der eindeutigen Tatbestandsbildung auf die Erfassung aller Fälle: der Gedanke eine Ehe zu führen, ist nicht rechtlicher Art, juristische Einheiten können auch ohne Mehrheitsbeteiligung ebenso intensiv zusammengefasst sein, usw. Aus der Sicht der Gerechtigkeit kann das Recht dies aber nur tun, wenn diese Fälle bezüglich ihrer Regelungswürdigkeit immerhin die Haupt-Fälle sind.

Was das Netzwerk angeht, so ließe sich meines Erachtens denken, rechtlich den Aspekt der Horizontalität herauszuheben, bringt er doch gegenüber allen andern, jeweils eine Leitung installierenden rechtlichen Organisationsformen, ein entscheidend neues Merkmal. Indessen geht namentlich die betriebswirtschaftliche Literatur ohne weiteres darüber hinaus - so weit, dass man den Eindruck gewinnt, es gehe beim jetzigen Stand der Forschung mehr um das Postulat eines irgendwie gearteten in-between zwischen

1 Richard Buxbaum (1993) »Is ,Network' a Legal Concept?«, 149 Journal of Institutional and Theoretical Economics, 698-705, 704. 
den bestehenden Ordnungsinstrumenten wie auch zwischen Ordnung und Nicht-Ordnung als um dessen positive Umschreibung ${ }^{2}$ - Netzwerk als der Zauber des Benennens des Unbekannten. Eher ist darum auf die neutralere Umschreibung der Sozialwissenschaften zu greifen, welche sich auf das beschränkt, was sicher feststeht, nämlich dass (soziale) Netzwerke durch Beziehungen zwischen Personen oder andern Handlungseinheiten konstituiert werden ${ }^{3}$. Das macht nun tatsächlich die Horizontalität seinerseits zu einer Eigenschaft des Gebildes.

Bis heute kennt dass Recht solche horizontale Gebilde nicht. Selbst die einfache Gesellschaft (Gesellschaft bürgerlichen Rechts), an die man zunächst in diesem Sinn denken könnte, kennt Gesellschafterbeschlüsse und Geschäftsführungsfunktionen, das will heißen: die Möglichkeit, für gewisse Personen einen rechtlich sanktionerten Willen verbindlich zu machen, also hierarchische Macht auszuüben ${ }^{4}$. Ein rechtslogisches Hindernis besteht dagegen allerdings nicht; eine auf Einstimmigkeit gegründete Organisation, in welcher mithin die Tätigkeit Einzelner nur der Vollzug des Beschlusses aller Glieder ist, lässt sich durchaus denken. Auch das würde aber keineswegs den Eintritt des Netzwerks als solchen ins Recht bedeuten. Vielmehr wäre es nur ein Netzwerk. Horizontalität bezeichnet lediglich die Eigenschaft einer Organisation, nicht eine Organisation selber. Angesichts des numerus clausus von Organisationsformen wäre der Tatbestand einer solchen Organisation spezifisch festzulegen. Wie kann das geschehen ${ }^{5}$ ?

\section{Die rhetorische Dimension}

Dass »Netzwerk« kein Rechtsbegriff ist, macht indessen nicht nur das Phänomen

2 Auch in der Betriebswirtschaftslehre ein Faszinosum, umschreibt diese das (Unternehmens-)Netzwerk etwa so: »...Organisationsform..., die die Koordinationspotentiale von Markt und Hierarchie (Organisation) in intelligenter Weise miteinander verknüpft und die sich infolge durch komplex-reziproke, eher kooperative denn kompetitive und relativ stabile Beziehungen zwischen mehr als zwei rechtlich selbständigen, wirtschaftlich jedoch mehr oder weniger abhängigen Unternehmungen auszeichnet « (Jörg Sydow und Bennet van Well (2003) »Wissensintensiv durch Netzwergorganisation«, in: Jörg Sydow (Hg.) Management von Netzwerkorganisationen, 3. Aufl., Wiesbaden: Gabler, 107-150, 113; übernommen etwa von Jens Aderhold (2004) Form und Funktion sozialer Netzwerke in Wirtschaft und Gesellschaft: Beziehungsgeflechte als Vermittler zwischen Erreichbarkeit und Zugänglichkeit, Wiesbaden: VS Verlag für Sozialwissenschaften (nach Jörg Sydow (1992) Strategische Netzwerke - Evolution und Organisation, Wiesbaden: Gabler, 78 ff.).

3 Schon Radcliffe-Brown, offenbar erster Verwender des Netzwerkbegriffs im soziologischen Kontext, gelangt dazu wie folgt: «The exchange ... is ... the result of, and at the same time ... a means of maintaining a certain structure, a network of relations between persons and collections of persons « (Alfred Radcliffe-Brown (1940) «On Social Structure", 70 Journal of the Royal Anthropological Institute of Great Britain, 1-12, 8; nach Aderhold (Fn. 2) 106; s. dort auch 120-134. Nicht anders die Netzwerkwissenschaft selbst, s. schon im Titel Albert-Laszló Barabási (2003) Linked, 2. Aufl., London: Penguin.

4 Eingehender Jean Nicolas Druey (2004) »Organisationsnetzwerke: Die ,schwebende< Rechtsform «, in: Marc Amstutz (Hg.) Die vernetzte Wirtschaft, Zürich: Schulthess, 123149, 127-129.

5 Dazu noch unten I. 6. 
(oben Zif. 1), sondern auch den Begriff selber nicht einfach irrelevant. Die Macht der Begriffe reicht weiter als nur das rationale Aufschließen von Gegebenheiten. Ihre Funktion kann nämlich nicht nur denotativ, sondern auch konnotativ sein, d.h. die Begriffe können aus Vorstellungen entstanden sein und Vorstellungen wachrufen, die keine Ableitung im Sinn des Subsumtions-Dreischritts (»Alle Frauen sind klug/Klothilde ist eine Frau/Also ist Klothilde klug«) erlauben. Es ist, was Umberto Eco semiotisch »die > offene< Logik der Signifikanten « nennt ${ }^{6}$.

In andern Worten kann die Entstehung eines Begriffs von zweierlei Art sein: Was neu daran ist, braucht nicht notwendig das reale Phänomen zu sein, das damit bezeichnet wird, sondern der Anlass kann nichts Anderes als das gesteigerte Bewusstsein und die erhöhte Aufmerksamkeit sein, die einer an sich alten Erscheinung entgegengebracht werden. So ist etwa die Selbstbezeichnung unserer Zeit als »Informationsgesellschaft « nicht nur der Reflex neuer Technologien, sondern, in Wechselwirkung damit, die gedankliche Wahrnehmung von Information als solcher, unabhängig von der Technik ihrer Verarbeitung. Insofern ist der Begriff weniger Beschreibung als Traktandum und hat den Reiz der ertragversprechenden Entdeckung.

So ist auch Netzwerk in hohem Maß zum (positiv besetzten) Reizwort geworden. Dabei verstärkt dieselbe Offenheit, die es rechts-technisch untauglich oder schwer handhabbar macht, diese Anziehungskraft und damit die rechts-politische Präsenz des Begriffs. Denn sie fördert die Möglichkeit, Idealvorstellungen daran festzubinden, und damit den Druck auf das Recht. Das Recht kann an solchen gesellschaftlichen Impulsen nicht vorbeileben. Zumindest als metarechtliche Gegebenheiten, als Entstehungsfaktoren von Rechtsnormen (auch etwa in Verträgen) muss es sie in der Auslegung berücksichtigen.

Die Chance des Eintritts ins Recht hat jedes Normgebilde zudem natürlich durch die Umdeutung. Was rechtliches Netzwerk sein will, kann vielleicht durch Unterlegung anderer Rechtsinstitute »gerettet « werden. Dies selbst dann, wenn das intendierte Netzwerk mangels Bestimmtheit nicht vollständig umsetzbar ist - sofern der Parteiwille darauf schließen lässt, dass es besser ist als vollständige Unwirksamkeit.

\section{Soziale und andere Netzwerke}

Netzwerke verbinden mithin alles, was verbunden werden kann. Namentlich können es sowohl Verbindungen von Personen oder aber von Objekten sein, und diese können ebenso konkreter wie abstrakter Natur sein. In den sozialen Netzwerken, welche Personen oder Personengruppen verbinden, einerseits, und den Objektverbindungen, andererseits, scheint sich Hauriou's Unterscheidung von »institution personnes« und »institution choses « abzubilden ${ }^{7}$.

Auch das Thema dieses Hefts könnte, rein sprachlich, auf die eine wie auf die andere Seite weisen. »Vertragsnetzwerke« würden dann ein soziales Netzwerk ansprechen, wenn die Verbindung vertraglich hergestellt wird, wenn also Personen sich ver-

6 Umberto Eco (2003) Einführung in die Semiotik, 9. Aufl., München: Fink, A. 3. IV. 1 betreffend poetische Botschaft; vgl. auch A. 5. zur Rhetorik. 
traglich zu einem Netzwerk zusammenschließen. Im Ausdruck »Vertragsnetzwerke« kann aber das Qualificans »Vertrag « auch das Objekt bezeichnen. Verbunden sind dann mehrere Verträge, und das ist, was das Thema hier meint.

Auf unserer Pfadsuche vom Netzwerk ins Recht stellt sich aber die Frage, ob die Wege sich hier trennen. Die Probleme sind in der Tat völlig verschieden. Sie sind größer und grundsätzlicher in Hinsicht auf die sozialen Netzwerke.

Soziale Netzwerke sind allgegenwärtig. Sie verbinden Menschen in verschiedenster Art; die Verbindung kann gleichsam alles von der eisernen Kette bis zum feinsten Spinnfaden sein. Das Recht kann auf keinen Fall alles erfassen, geschweige denn in eine harmonische Ordnung bringen. Es hat eine Auswahl zu treffen, welchen sozialen Körpern es sich widmen will. Es tut dies mit dem Grundsatz des numerus clausus der rechtlichen Organisationsformen. Dies blendet nicht nur sehr viele tatsächliche Verbindungen aus, sondern schirmt auch das Recht gegen Irritation durch das Phänomen ab. Denn die Initiative wird auf die Rechts-Klienten verschoben; sie haben durch Wahl eines der Modelle die Rechtsförmigkeit ihrer Assoziation kundzutun.

Damit ist ein weiteres Stück Antwort auf die Frage der Naturnotwendigkeit gegeben: Durch seine Natur ist das Recht außerstande, das (soziale) Netzwerk als solches zu einem Rechtstatbestand zu machen. Es lässt sich auch so sagen: Recht kann nicht Verbundenheit per se regeln, sondern setzt erst bei der Qualität, also der Eigenart der jeweiligen Verbindung an.

Die verbundenen Objekte dagegen machen dem Recht den Zugang sehr viel leichter. Es geht nicht darum, das Netzwerk als ein Phänomen für sich zu fassen, sondern einfach die Verbundenheit der Elemente zu erkennen. Gewiss: auch solche Elemente können zusammen ein Ganzes bilden, das seinerseits wiederum nach außen als Einheit auftritt. Die Atome finden sich im Molekül, die Moleküle in einem Stoff, usw. Doch das Molekül ist nicht im gleichen Sinn ein Netzwerk von Atomen wie es das soziale Netzwerk in Hinsicht auf seine menschlichen Glieder ist. Beim Zweitgenannten sind sowohl das Ganze wie die Teile Einheiten; das Ganze setzt die Teile nicht nur als Teile, sondern als Träger, als aktive Konstituenten voraus. Das Objekt-Netzwerk dagegen duldet keine Symbiose von Ganzem und Teilen; Einheit ist das Eine oder das Andere, je nach Blickweise.

Letzteres ist der Fall auch der Vertragsnetzwerke, wie sie dieser Band und frühere juristische Untersuchungen behandeln. Fragestellung ist die Einwirkung der Vernetzung auf die Einheiten, mithin den einzelnen Verträgen. Entsprechend geht die Beurteilung von den Elementen aus; zu untersuchen ist, ob sie aus sich heraus auf andere Netzeinheiten verweisen. Die Frage ist von vornherein juristisch und damit ohne weiteres eine rechtliche Binnenangelegenheit.

7 Maurice Hauriou (1925) »La théorie de l'institution et de la fondation. Essai de vitalisme social «, in deutscher Übersetzung (1965) »Die Theorie der Institution: und zwei andere Aufsätze«, in: Roman Schnur (Hg.) 5 Schriften zur Rechtstheorie, Berlin: Duncker \& Humblot, 35 . Die Verunsicherung, die Haurious Begriff von der institution-chose hinterlassen hat, hängt damit zusammen, dass er diesen dann auf die Rechtsnorm als einem Anwendungsfall konzentriert hat. 


\section{Die sozialen Netzwerke sind alle organisationaler Natur}

Für die sozialen Netzwerke erhebt sich nun die Frage, durch welche Tür sie ins Recht eintreten. Bei den Sach-Netzwerken erübrigt sich diese Frage, weil dieser Typ von vornherein an eine Disziplin gebunden ist: die Bindung von Atomen ist eine physikalische, diejenige von Körperzellen eine biologische und eben von Verträgen eine juristische.

Soziale Netzwerke sind immer Organisationen. Denn sie stellen ein bloßes Wirkungs-Potenzial dar, was sich im Bereich menschlicher Zweckgebilde als Merkmal von Organisationen bezeichnen lässt. Sie sind auf Leistung angelegt, lassen sich aber von den einzelnen Leistungsvorgängen gedanklich trennen, weil sie die Dimension der Dauer aufweisen ${ }^{8}$.

Nun hat sich aber, offenbar von der Betriebswirtschaftslehre kommend, die Sicht verbreitet, wonach Organisation nur der eine Aspekt des Netzwerkphänomens sei. Das Netzwerk wird dahin charakterisiert, dass es »zwischen Hierarchie (Organisation) und Markt« positioniert sei. Lassen wir offen, wie sehr die Betriebswirte selber solchen Umschreibungen treu bleiben ${ }^{9}$. Jedenfalls reicht die Umschreibung für juristische Zwecke nicht aus. Denn Kriterien, die sich ausschließen, lassen sich nicht einfach mischen, ohne jedem von ihnen seinen eigenen Bereich zuzuweisen, oder sie in eine Hierarchie zueinander zu bringen - kurz: sie in eine Ordnung zu stellen.

Hier trennt sich der Weg von demjenigen von Gunther Teubner, dem Vordenker in dieser Materie ${ }^{10}$. Er sieht keinen Anlass für das Recht, das außerrechtlich entstandene Paradoxon nicht auf sich beruhen zu lassen, sondern im Gegenteil soll es dieses angesichts seiner wirtschaftlichen Notwendigkeit fördern ${ }^{11}$. Sehr zu recht hat er dabei die vielen Situationen von Doppelbindungen im Auge, die einzelnen Personen und Einheiten die Austragung von Widersprüchen in den Vorgaben gleichsam »in der eigenen Brust « auftragen ${ }^{12}$. Ich sehe indessen nicht, was das Recht bieten kann, wenn es sich

8 Die verbreitete, zumindest partielle Kennzeichnung des Netzwerks als Hierarchie (dazu noch unten im Text dieser Zif.) besagt in dieser Hinsicht nichts Anderes.

9 Sydow (Fn. 2) setzt selber ein anderes durch das Netzwerk zu synthetisierendes Gegensatzpaar auf, wenn er die Elemente als rechtlich autonom, wirtschaftlich aber abhängig beschreibt. Abgesehen davon, dass dies die Merkmale des Konzerns sind, und schon darum für das Netzwerk wohl wesentlich zu kurz greifen, ist damit nichts über die Mischung verschiedener Strukturen im Netzwerk ausgesagt, sondern lediglich eine Mehrheit von Sichtweisen auf das Netzwerk genannt.

10 Ich stütze mich in erster Linie auf Gunther Teubner (2004) Netzwerk als Vertragsverbund: Virtuelle Unternehmen, Franchising, Just-in-time in sozialwissenschaftlicher und juristischer Sicht, Baden-Baden: Nomos, aber auch ders. (2004a) »Coincidentia oppositorum: Das Recht der Netzwerke jenseits von Vertrag und Organisation«, in: Marc Amstutz (Hg.) Die vernetzte Wirtschaft - Netzwerke als Rechtsproblem, Zürich: Schulthess, 11-42, sowie ders. (2001) »Das Recht hybrider Netzwerke«, 165 ZHR, 550-575, und Marc Amstutz und Gunther Teubner (2006) »Editorial «, in diesem Heft oben.

11 Teubner (2004) (Fn. 10) insb. 79-88.

12 Jean Nicolas Druey (2006) »Interessenkonflikte «, in: Charlotte Baer (Hg.) Verwaltungsrat und Geschäftsleitung (76 St. Galler Studien zum Privat-, Handels- und Wirtschaftsrecht), Bern : Haupt, 59-77, 69-72. 
damit befasst. Substituiert es sich im Entscheid, so muss es den Widerspruch auflösen; substituiert es sich nicht, so entsteht ein entsprechender Bereich freien, mangels Kriterien auch nicht rahmenmäßig überprüfbaren Ermessens, d.h. von Seiten des Rechts würde eine Normsetzungsdelegation stattfinden.

Nun besagt dies freilich nicht mehr, als dass rechtlich die Frage ob Vertrag oder Organisation in der Tat das experimentum crucis ist. Denn von vornherein kann nur in der Organisation von Schranken der Rechtsetzungsdelegation die Rede sein, weil Organisationsrecht immer auch Dritte betrifft, die an der internen Rechtssetzung nicht beteiligt sind. Für Verträge gilt dagegen die Vertragsfreiheit; in ihr liegt per se die Delegation der Rechtsetzung. Die Frage, was von beidem nun rechtlich vorliegt, ist also unausweichlich und stellt sich erbarmungslos als ein Entweder/Oder dar.

Und doch kennt auch das Recht durchaus die Mischung, nur heißt »mischen « etwas Anderes, so wie sie für die Collagetechnik anders verwirklicht wird als in der Ölmalerei. Das Recht ist auf der Seite der Collage, es arbeitet mit Schichten, die übereinander liegen und durchscheinen lassen können. In diesem Sinn reduziert sich das Unumgängliche auf die Bestimmung der rechtlichen Oberfläche, und ihr gilt der hier gezogene Schluss, dass das rechtliche Bild des Netzwerks die Organisation ist. Damit ist nichts Anderes gesagt, als dass Zuständigkeiten und Ablaufweisen für noch nicht festgelegte Benutzungen bestimmt werden. Struktureigenschaften wie Hierarchie oder Markt sind damit noch nicht berührt. Das sind außerjuristische Begriffe; verboten ist darum m.E. die Gleichsetzung von »Hierarchie « mit »Organisation«, wenn letztgenannte im juristischen Sinn gemeint sein soll. Denn jede juristische Organisation mischt, enthält mithin sowohl Hierarchie wie Markt. Das ist nicht typisch für Netzwerke, es sei denn, alle Organisationen seien Netzwerke. In jeder Unternehmung, und sei sie noch so straff hierarchisch geführt, sind interne Austauschverhältnisse, ist das »do ut des « ein vitales Element, namentlich im informationellen Bereich.

Auf dieser sekundären Ebene kann selbstredend auch umgekehrt ein Vertrag Zuständigkeiten und Abläufe regeln, aber ebenso selbstverständlich nur nach den Gesetzen auf der Primärebene, d.h. unter den Parteien, welche durch den Vertrag eine Grundlage für ihr Verhältnis gelegt haben. Die dargelegte Betrachtungsweise verweigert sich, und da liegt vielleicht der entscheidende Punkt in der juristischen Theoriebildung, einer quantitativen Argumentation, wonach das Netzwerk durch ein »starkes«, »deutliches « oder wie immer quantitativ erhebliches Auftreten der beiden Elemente als dritte mittlere Möglichkeit gekennzeichnet wäre. Dies muss so sein, solange es die dritte Form im Recht nicht gibt, d.h. als nicht auch die sachgerechten Rechtsfolgen bezeichnet sind, welche sich an die Netzwerkeigenschaft knüpfen.

\section{Gewalt als Element - auch und just des Privatrechts}

Privatrecht hat die Aufgabe, wirtschaftliche Transaktionen vorzubereiten, indem es ihnen vorgeformte Elemente zur Verfügung stellt, welche die Verhandlungs- und Durchführungskosten zu senken erlauben. Akzeptiert man diese Funktion, so hat es zur Folge, dass die Übersetzung vorfindlicher außerrechtlicher Verbindungen in rechtliche Gefäße vom Recht nach Möglichkeit erleichtert werden muss. Vagheit und Wi- 
dersprüchlichkeit sind weder aus der Sicht des Gesetzgebers noch derjenigen des Richters ein Grund, diese Hilfe a priori zu versagen. Diese Funktion impliziert aber, weil die Überführung nicht $1 \mathrm{zu} 1 \mathrm{möglich}$ ist, dass den Parteivorstellungen ein Stück weit, nämlich soweit das Interesse des Ganzen es rechtfertigt, Gewalt angetan werden muss. Die Prokrustes-Rolle ist mithin dem Privatrecht, als eine negotiorum gestio, just kraft seiner Dienstleistungsaufgabe inhärent.

Der Punkt, an welchem die Ausblendung von Außerrechtlichem kontraproduktiv wird, ist dann erreicht, wenn davon Wesentliches der Parteivorstellungen betroffen ist. Das große, weit über Netzwerke hinausreichende, aber auch für sie typische Problem der Rechtsschöpfung und -anwendung liegt darin, dass die Herstellung von Klarheit und Widerspruchslosigkeit keineswegs zu diesen Vorstellungen gehören muss, sondern im Sinn Franz Kleins eine »Lebenslüge $«{ }^{13}$ darin enthalten sein kann, deren Aufdeckung nicht gewünscht ist. Klarstellung und Geradebiegen in der Verrechtlichung ist darum praktisch immer auch ein Popularitätsverlust einer Institution. Ich meine aber, dass dies eine notwendige Konsequenz ist, die nur durch Verzicht a priori auf die rechtliche Erfassung (namentlich in den frühen Phasen einer Entwicklung) vermieden werden kann; ein Krummbiegen des Rechts im Sinn des »Entgegenkommens« ist m.E. nicht denkbar.

\section{Das Recht als Netz, für Netzwerke}

Das Ergebnis des Vorangehenden besagt, dass in einem ganz andern Sinn als sonst verwendet auch das Recht als ein »Netz der Netze « zu bezeichnen ist. Freilich liegt darin ein Wortspiel, doch Wortspiele sind ernst zu nehmen. »Netz« hat dabei nicht die Bedeutung der $B e$-schreibung (eines Phänomens) als Verbindung bzw. Verbindungsverbindung, sondern als $Z u$-schreibung (eines Zwecks) als Institution des Auffangens. Soweit Netzwerke auf das Recht angewiesen sind (es wird davon ausgegangen, dass es zumindest teilweise der Fall ist), relativiert sich ihre Selbstqualifikation als Netzwerk, weil sie auf das Begriffssubstrat angewiesen sind, das vom Recht unter diesem Stichwort gespeichert ist.

Nicht nur als Metapher, sondern als deduktionsfähige abstrakte Charakterisierung sind Netze durchlässig. So ist es auch das Normenkonglomerat, das sich als Recht bezeichnet. Und wir haben gesehen, dass in der Tat Vieles von dem, was außerjuristisch als »Netzwerk « gilt, durch das juristische Netz durchfällt. Das Recht ist heute keiner Bewältigung einer horizontalen Organisation fähig. Es kann auch keine sozialen Institutionen aufnehmen, nur weil sie »in action«, also gelebt, sind; es muss sie in eine folgerichtige Ordnung bringen können.

13 Franz Klein (1914) »Die wirtschaftlichen und sozialen Grundlagen des Rechtes der Erwerbsgesellschaften«, in: 7 Vorträge und Schriften zur Fortbildung des Rechts und der Juristen, Berlin: Franz Vahlen, 88: «wohltätige Illusionen«, »sorgfältig erhaltene ... Selbsttäuschungen « als »Mittel der Politik des Lebenkönnens«; dazu Peter Doralt und Susanne Kalss (Hg.) (2004) Franz Klein - Vorreiter des modernen Aktien- und GmbH-Rechts, Wien: Manz; Jean Nicolas Druey (2004) »Franz Klein weiterdenken«, in: ibidem, 139-160, $145 \mathrm{f}$. 
Das Recht muss sich aber, soweit die Verrechtlichung gewünscht ist, nach seinem Selbstverständnis dafür zur Verfügung stellen. Der Rechtsstaat muss insofern auch über seine Orakelfunktion hinaus ein aktives Auge auf die Szenen werfen, die einen wichtigen Bedarf nach seiner Hilfe indizieren, dies auch im Sinn der Mitwirkung bei der Erfassung der Phänomene im Licht dieses Bedarfs.

Doch das Recht bildet nicht nur ein Netz für, sondern auch gegen Netzwerke. Hier möchte der vorliegende Beitrag eine Diskussion mit Gunther Teubner aufnehmen. Nach der oben dargelegten Meinung muss das Paradox aus dem Begriff, dem es innewohnt, vor seinem Eintritt ins Recht ausgefiltert werden. Dies trotz allem Wert, trotz der unleugbaren Fruchtbarkeit, ja Notwendigkeit von Paradoxen in der sozialen und überhaupt geistigen Welt. Vielleicht bin ich gar der Bösgläubigkeit zu zeihen, wenn ich selber vom »Eintritt« oder von der »Aufnahme« des Netzwerkbegriffs ins Recht spreche. Denn dort liegt es im Grunde schon: Das Recht nimmt nicht Begriffe auf, sondern verwendet sie als Tatbestandselement für Rechtsfolgen. Eine Erscheinung, deren Wesen das Schillern (auch) hinsichtlich der Rechtsfolgen ist, scheint mir für das Recht (noch) untauglich.

Damit ist nichts gegen die Möglichkeit gesagt, Gegensätze im Sinne Teubners rechtlich durch Doppelbindungen aufzufangen. Ich meine, dass in der Funktion, und zwar vielleicht in jeder Funktion, der Schmelztiegel für solche Mehrfachorientierungen angelegt ist. Denn abstrakt formuliert zeichnet sich eine Funktion durch Zuweisung eines Wirkungsbereichs und nicht eines Ziels aus, kann also die Bestimmung des Ziels in sich selbst enthalten. Das ist von kaum überschätzbarer praktischer Bedeutung, hat doch jeder Beamte (»Kundenfreundlichkeit« v. Kontrolle der Bürger), jeder Lehrer (Schülerförderung v. Vollzug der Schulordnung), aber auch jeder Manager (verkleistert unter dem »shareholder's value«) und das Unternehmen selber (z.B. Umweltbelastung v. Umweltverantwortung) solche Gegensätze autonom zu absorbieren, und das aktuelle Problem dürfte bei der »Feigheit« liegen, mit welcher solche Konflikte von den Normsetzern in die Funktionen delegiert werden. Damit ist aber auch die rechtliche Unverdaubarkeit der Mehrfachbindung festgestellt. Die Normsetzungsdelegation, die damit stattfindet, weist insoweit das Thema wieder zum Recht hinaus. 